\title{
Title: Nuclear Magnetic Resonance Imaging with Hyper-Polarized Noble Gases
}

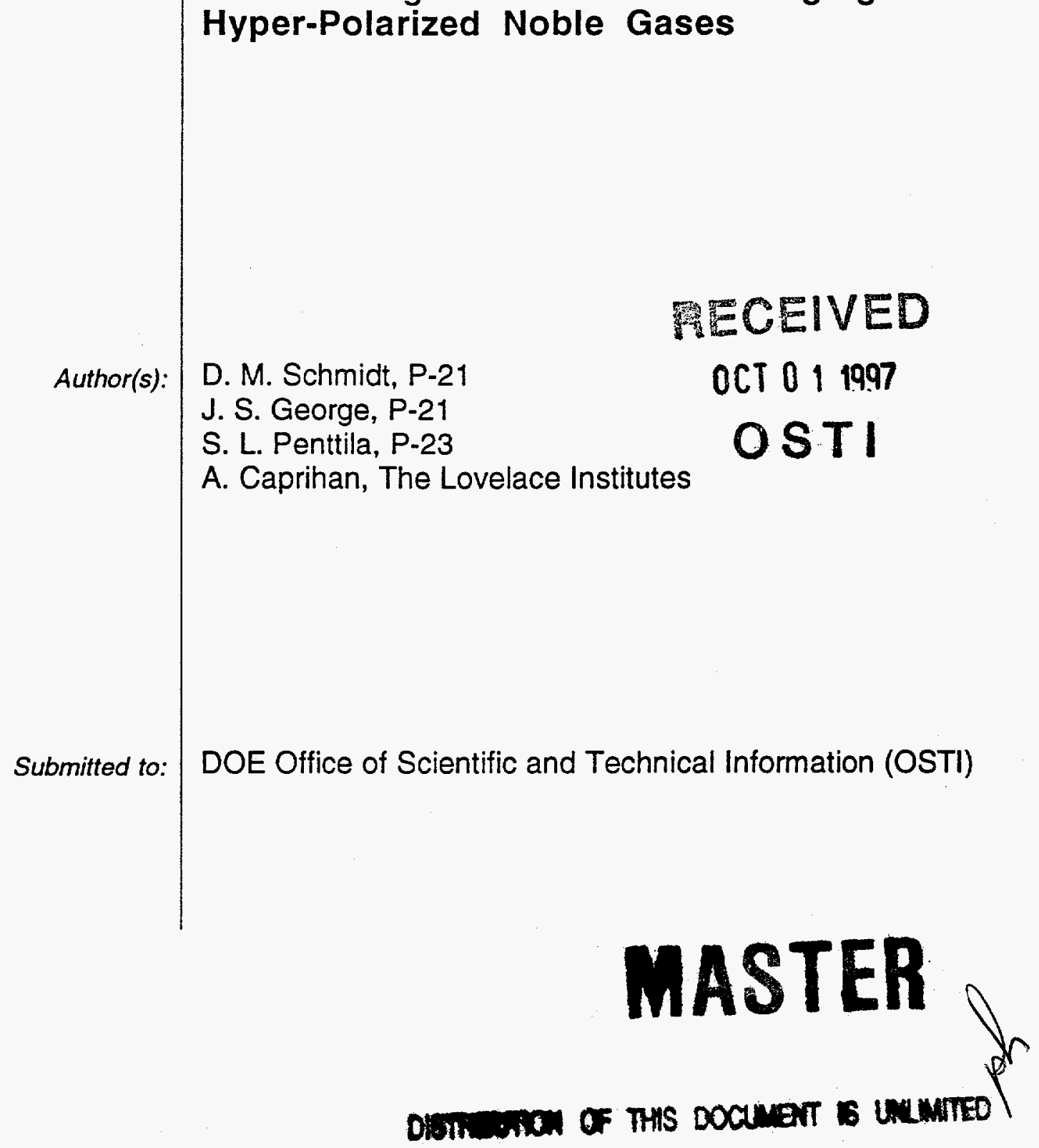

\section{Los Alamos}

NATIONAL LABORATORY

Los Alamos National Laboratory, an affirmative action/equal opportunity employer, is operated by the University of Califomia for the U.S. Department of Energy under contract W-7405-ENG-36. By acceptance of this article, the publisher recognizes that the U.S. Government retains a nonexclusive, royaltyfree license to publish or reproduce the published form of this contribution, or to allow others to do so, for U.S. Government purposes. Los Alamos Nationa Laboratory requests that the publisher identity this article as work performed under the auspices of the U.S. Department of Energy. Los Alamos National Laboralory strongly supports academic freedom and a researcher's right to publish; as an institution, however, the Laboratory does not endorse the viewpoint of a publication or guarantee its technical correctness. 


\section{DISCLAMIER}

Portions of this document may be illegible in electronic image products. Images are produced from the best available original document. 


\section{DISCLAIMER}

This report was prepared as an account of work sponsored by an agency of the United States Government. Neither the United States Government nor any agency thereof, nor any of their employees, make any warranty, express or implied, or assumes any legal liability or responsibility for the accuracy, completeness, or usefulness of any information, apparatus, product, or process disclosed, or represents that its use would not infringe privately owned rights. Reference herein to any specific commercial product, process, or service by trade name, trademark, manufacturer, or otherwise does not necessarily constitute or imply its endorsement, recommendation, or favoring by the United States Government or any agency thereof. The views and opinions of authors expressed herein do not necessarily state or reflect those of the United States Government or any agency thereof. 


\title{
Nuclear Magnetic Resonance Imaging with Hyper-Polarized Noble Gases
}

\author{
David M. Schmidt*, John S. George, and Seppo I. Penttila \\ Los Alamos National Laboratory \\ Arvind Caprihan \\ The Lovelace Institutes
}

\begin{abstract}
This is the final report of a six-month, Laboratory Directed Research and Development (LDRD) project at Los Alamos National Laboratory (LANL). The nuclei of noble gases can be hyper polarized through a laser-driven spin exchange to a degree many orders of magnitude larger than that attainable by thermal polarization without requiring a strong magnetic field. The increased polarization from the laser pumping enables a good nuclear magnetic resonance (NMR) signal from a gas. The main goal of this project was to demonstrate diffusion-weighted imaging of such hyper-polarized noble gas with magnetic resonance imaging (MRI). Possible applications include characterizing porosity of materials and dynamically imaging pressure distributions in biological or acoustical systems.
\end{abstract}

\section{Background and Research Objectives}

In conventional nuclear magnetic resonance (NMR) and magnetic resonance imaging (MRI), the signal is derived from nuclei that are thermally polarized in the presence of a large magnetic field, usually from a superconducting solenoid. The degree of polarization is proportional to the magnetic field strength. In contrast, the nuclei of noble gases can be hyper polarized through a laser-driven spin exchange to a degree many orders of magnitude larger than that attainable by thermal polarization, without requiring a strong magnetic field [1]. Whereas thermally polarized gases are traditionally very difficult to image because of their low density, the increased polarization from optical pumping more than makes up for the low density, allowing one to obtain a good NMR signal from a gas. While others have demonstrated the advantages of a large signal [2] and the ability to transfer polarization to other nearby nuclei [3] using hyper-polarized noble gases, we wanted to demonstrate some novel advantages using diffusion.

\footnotetext{
* Principal Investigator, e-mail: dschmidt@lanl.gov
} 


\section{Importance to LANL's Science and Technology Base and National R\&D Needs}

The unique characteristics of NMR with hyper-polarized noble gases have the potential to dramatically improve current NMR and MRI applications and to create important, qualitatively new applications both by increasing sensitivity and by removing the restrictions associated with a large magnetic field. Examples include truly portable, much less expensive NMR and MRI instrumentation for remote geophysical and medical diagnostics, improved characterization of porosity and fractures for modeling hazardous waste transport, new and improved methods for measuring surface properties in material science, and novel methods for molecular structure determination.

\section{Scientific Approach and Accomplishments}

We used a polarization apparatus originally constructed for nuclear physics experiments at the Los Alamos Meson Physics Facility (LAMPF) in combination with the MRI system at The Lovelace Institutes. A cylinder of $3 \mathrm{He}$ gas was laser-polarized using our apparatus at The Lovelace Institutes in the external magnetic field of the imaging magnet, at a distance of about 2 meters. After a few hours of polarization, the cylinder was moved into the bore of the magnet for imaging. Figure 1 shows an MRI image of $3 \mathrm{He}$ gas at 7 atm pressure hyper polarized by optical pumping. The cylinder containing the gas is about $7.5 \mathrm{~cm}$ long and $2.5 \mathrm{~cm}$ in diameter. This is a projection of the three-dimensional (3-D) cylinder onto a 2-D image,ich is why the outer layers have a lower signal.

It is unusual for a medium to have both a strong NMR signal and a large diffusion coefficient. We exploited this fact in two different experiments. First, we inverted the polarization in a thin central section of the cylinder and made a series of 1-D images of the cylinder over time in order to see the diffusion of the two populations of nuclei (one with polarization "up" and one with polarization "down"). Figure 2 shows the series of 1-D images of the $3 \mathrm{He}$ gas in the cylinder over a period of 5 seconds, one image every 0.2 seconds. The polarization in the central portion of the cylinder is being filled in by other nuclei diffusing into this section, rather than by thermal re-polarization which is what would happen in water, for example.

Using a very simple model, we measured the 3 He diffusion coefficient with this data. A delta-function spike in density will, through diffusion, form a density profile that 
is Gaussian whose width is proportional to the diffusion coefficient and the time over which diffusion has taken place. We therefore modeled each 1-D image by convoluting the first 1-D image with a Gaussian whose width was proportional to a candidate diffusion coefficient times the time interval separating the two images. We then searched for the value of the diffusion coefficient which minimized the error between the predicted and measured values. The result was a diffusion coefficient of $D=12.3+-0.2 \mathrm{~mm}^{2} / \mathrm{s}$. A comparison of the best-fitting model with the data is shown in Figure 3.

In the second experiment we constructed diffusion-weighted images of the gas. A diffusion coefficient was calculated at each point in the image by taking the ratio of the image intensities with and without a previous magnetic field gradient in place. With a gradient, the signal will diminish because of diffusion. Knowing the strength and duration of the gradient allows the diffusion coefficient to be determined.

Two 1-D diffusion images of the gas are shown in Figure 4. The plot on the left is with the gas at thermal equilibrium at room temperature. The diffusion coefficient away from the ends of the cylinder matches that measured earlier by a completely different diffusion-inflow method. The lowering of the diffusion coefficient near the walls of the cylinder is due to the walls restricting the diffusion in this area. The plot on the right shows a diffusion image when the cylinder had a thermal gradient produced by holding the right hand end of the cell in a liquid nitrogen exhaust plume. For an ideal gas the diffusion coefficient is proportional to the square root of the absolute temperature. The thermal gradient is nicely represented by the diffusion gradient in this image. Again, the lowering of the diffusion coefficient at the ends is due to restricted diffusion.

We have demonstrated some unique aspects of diffusion when using hyperpolarized gas in MRI. Just as the relaxation parameters T1 and T2 are used to highlight differences in physical environments in NMR and MRI, we feel that the diffusion coefficient $D$ can also be used to highlight environmental differences when using hyperpolarized noble gases in NMR and MRI. Whereas T1 and T2 are directly affected by the electro-magnetic properties of the local environment, $D$ is affected by the physical parameters of volume, temperature and pressure. Possible applications include characterizing porosity of materials and dynamically imaging pressure distributions in biological or acoustical systems. 


\section{References}

[1] Bhaskar, N.D., Happer, W., McClelland, T., Phys. Rev. Lett. 49, 25 (1982). Happer, W., Miron, E., Schaefer, S., Schreiber, D., van Wijngaarden, W.A., Zeng, X., Phys. Rev. A 29, 3092 (1984).

[2] M.S. Albert, G.D. Cates, B. Driehuys, W. Happer, B. Saam, C.S. Springer, Jr., A. Wishnia, Nature 370, 199 (1994). H. Middleton, R.D. Black, B. Saam, G.D. Cates, G.P. Cofer, B. Guenther, W. Happer, L.W. Hedlund, G.A. Johnson, K. Juvan, J. Swartz, Magn. Reson. Med. 33, 271 (1995).

[3] G. Navron, Y.-Q. Song, T. Room, S. Appelt, R.E. Taylor, A. Pines, "Enhancement of Solution NMR and MRI with Laser-Polarized Xenon," Science, 271, 1848 (1996). 


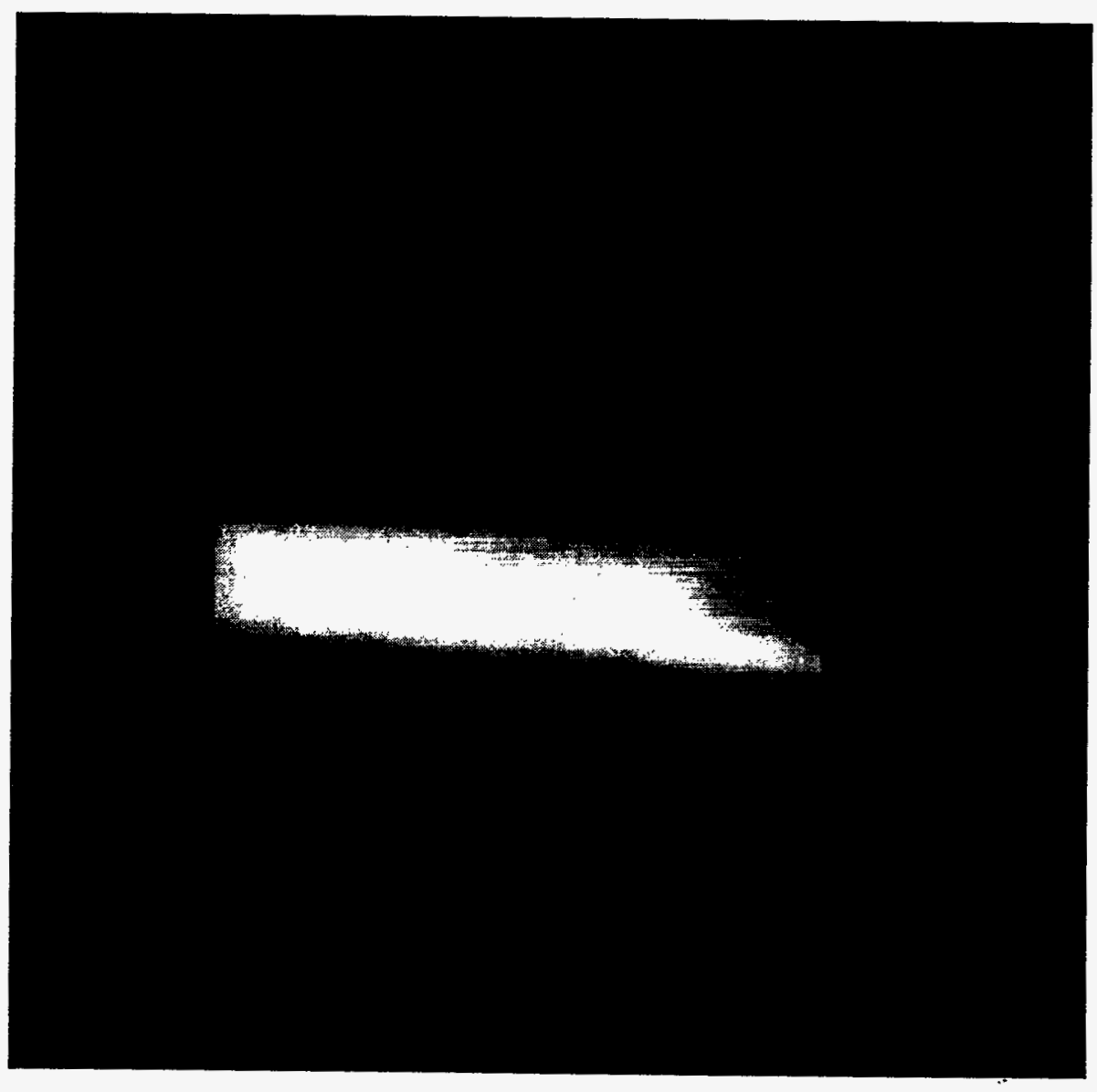

Figure 1. This figure shows an MRI image of $3 \mathrm{He}$ gas at 7 atm pressure hyper polarized by optical pumping. The cylinder containing the gas is about $7.5 \mathrm{~cm}$ long and $2.5 \mathrm{~cm}$ in diameter. This is a projection of the 3-D cylinder onto a 2-D image, which is why the outer layers have a lower signal. 


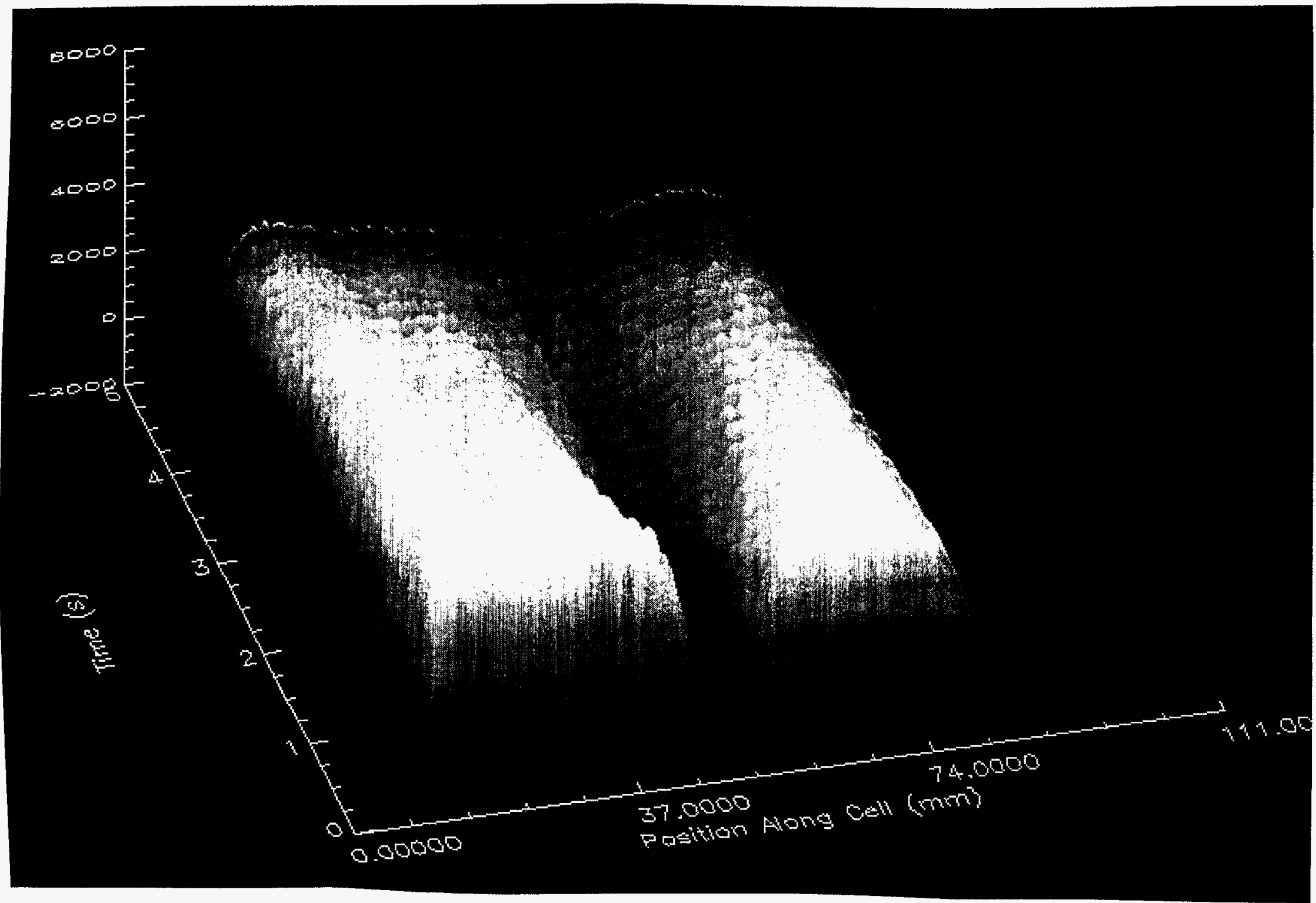

Figure 2. This figure shows a series of 1-D images of the $3 \mathrm{He}$ gas in the cylinder over a period of 5 seconds, one image every 0.2 seconds. The polarization in the central portion of the cylinder is being filled in by other nuclei diffusing into this section, rather than by thermal re-polarization, which is what would happen in water, for example. 


\section{Comparison of Fit to Data \\ $\mathrm{D}=12.3 \pm 0.2 \mathrm{~mm}^{\wedge} 2 / \mathrm{s}$}
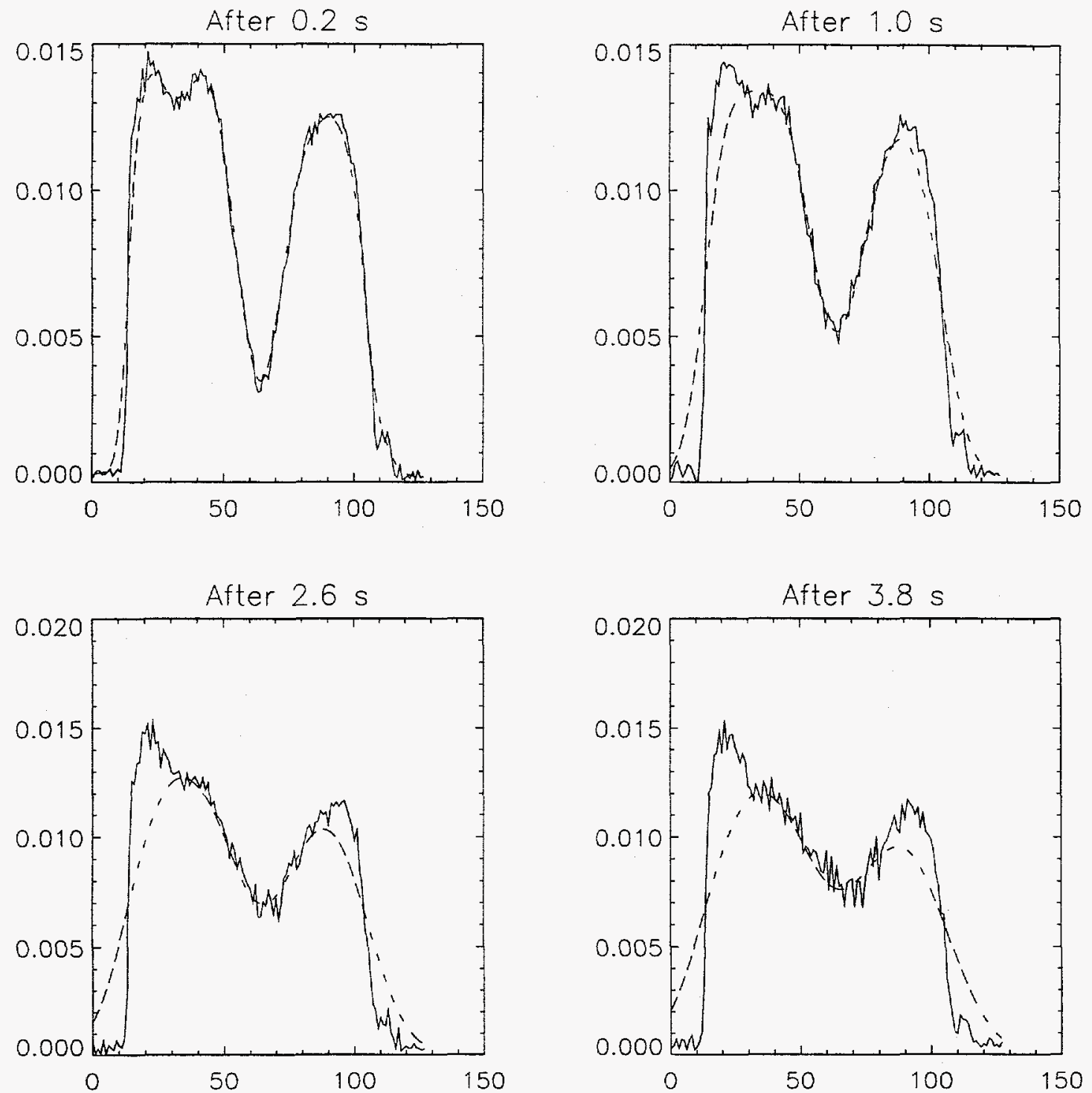

Figure 3. The data (solid line) and predicted values (dashed line) are compared for 4 different images for the best-fit value of the diffusion coefficient (shown at top) in the diffusion inflow experiment. The model used does not account for the walls of the cell. This does not affect the central portions of the images, however, which is what was used in determining the goodness-of-fit. 


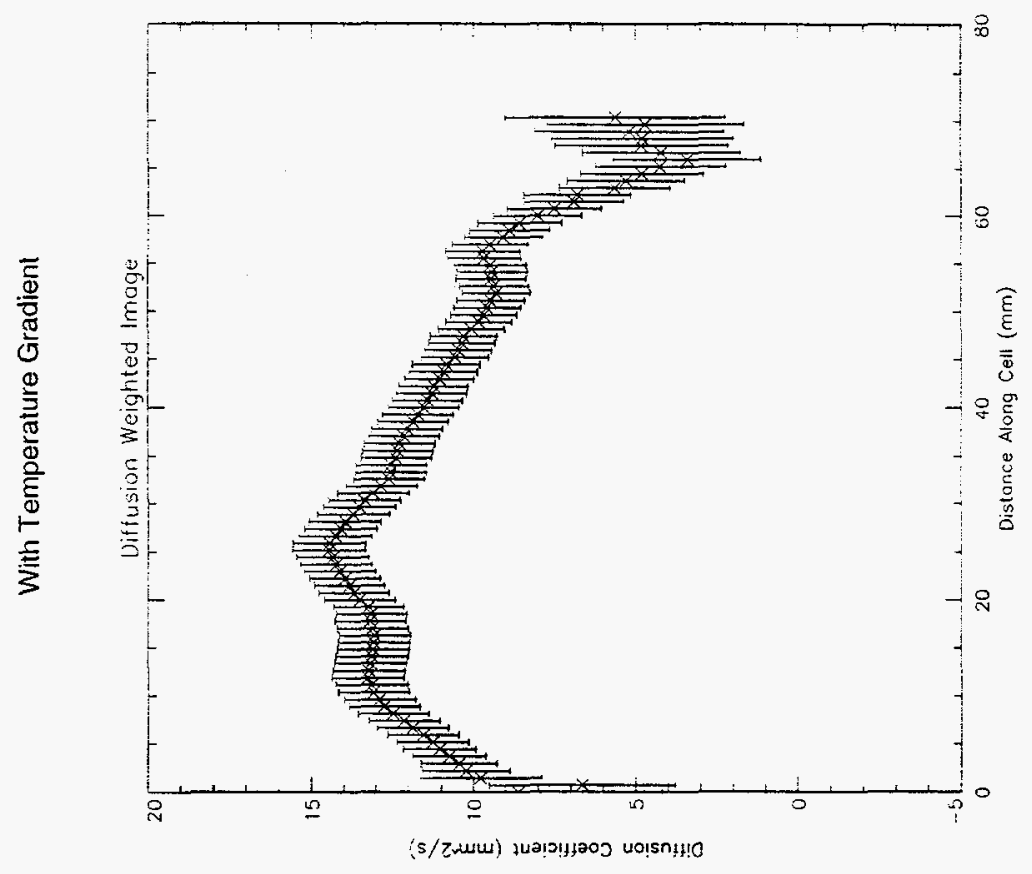

殉

폭

㤩

그

हี

올

政

茄司

크

들

3

.

응

응

झ

ธี

흥형

定究

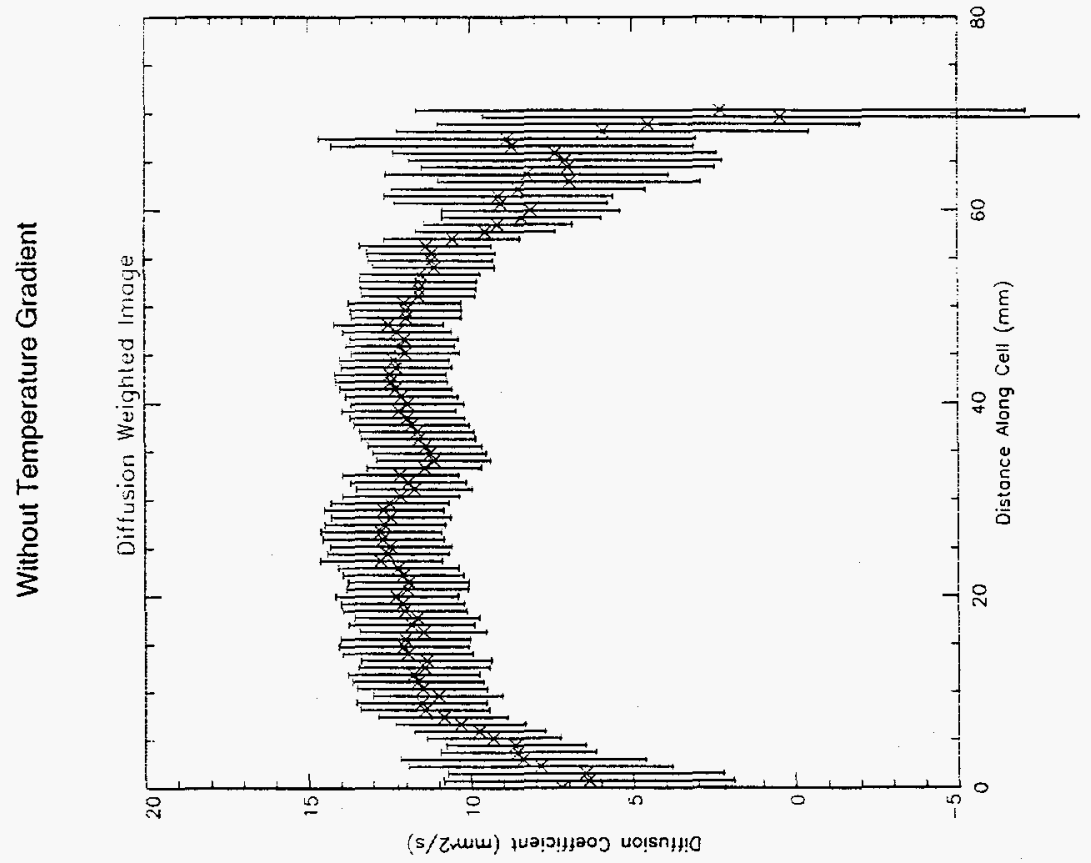

을

速罗

을

可

的氠

토

은

送

总完

马्ठ등

등

安运

\&

울융

氙逮

豆

I

를

$\$$

늘

$\checkmark$ 苋

ษ

5

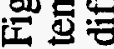

$\infty$ 